\title{
Capacity Building on Heat Balance in Electrolysis of Aluminium
}

Sobah AbbasPetersen ${ }^{1}$, Manuel Oliveira ${ }^{1}$, Kristin Hestetun ${ }^{2}$, Gunn Iren Müller ${ }^{2}$, Stein O. Wasb $\varnothing^{3}$, Victoria Koritzinsky ${ }^{4}$, Anne Lise Waal ${ }^{4}$

${ }^{1}$ SINTEF Digital, Trondheim, Norway, E-mail

\{sobah.petersen, manuel.oliveira\}@sintef.no

${ }^{2}$ Hydro Primary Metal Technology, Norway,

\{Kristin.Hestetun, Gunn.Iren.Muller\}@hydro.com,

${ }^{3}$ Cybernetica AS, Trondheim, Norway, stein.wasbo@cybernetica.no,

${ }^{4}$ Attensi AS, Trondheim, Norway, \{victoria.koritzinsky, anne.lise.waa\}@attensi.com

\section{Abstract}

Designing games for learning in the workplace is challenging in many ways. Within the process industry, the aluminium production cells are harsh environments that are difficult to instrument and pose domain related challenges, which make effective training difficult to achieve. In particular, cognitive challenges arise due to the slow nature of the physical process that disconnects the operators' actions and causality. The complex chemical process demands a high level of understanding of the parameters and their complex interdependencies. Additional challenges are related to representing an appropriate level of detail and precision of the workplace, including integration with existing tools. This paper describes the digital simulation game designed and developed for the operators in aluminium plants to master the cognitive skills required for Heat Balance in the aluminium production cells. A co-design process was adopted from the initial inception of the idea, development and evaluation. The paper will discuss and present the co-creation artefacts used, namely a paper prototype, hybrid prototype of the game along with the output from iterative development cycles. This work is conducted within the research and innovation project, Accelerated Learning for Technology Transfer, with the Norwegian company Hydro.

Keywords: Cognitive Challenges, Workplace Learning, Paper prototypes, Game Design, Dynamic Process Model

\section{Introduction}

The Norwegian industrial project Accelerate Learning Through Technology (ALTT) aims to build human capacity at Hydro, one of the largest aluminium production companies [1]. As a consequence of automation, operators of the aluminium plants are required to understand the different parameters and their complex inter-dependencies that are critical to ensure Heat Balance Control in the aluminium production cells. Due to the very nature of the chemical process, there is a need to cognitively build a mental model that facilitates an operator to diagnose the state of an aluminium production cell and anticipate and predict the best course of action to maintain the cell's stability, with minimal available information. Most of the operators learn about Heat Balance Control through practice and working and by observing and following instructions from their more experienced peers [2]. They follow detailed textual descriptions of Standard Operating Procedures (SOPs), but often lack a deep understanding of why they are doing something and, thus, the consequences of their actions. Such competences take several years to develop, with different production plants differing in their operating philosophies, which affect the reasoning strategies for decision making. 
Games have been described as an appropriate means of supporting learning [3]; particularly learning by doing $[4,5]$. Games provide a safe and cost-efficient simulation environment for learners to try different situations and explore and experiment, thus reducing the numbers of years it may take an operator to master the skills and competences that are required to operate an aluminium production cell efficiently. This paper describes the use of a digital simulation game to support the operators master heat balance in aluminium production.

This learning domain, as many other industrial situations, pose a variety of challenges that need to be addressed by game designers and designers of learning technologies. One of the main challenges is the representation of the industrial setting with an appropriate level of resemblance to the workplace, referred also as the simulation level [6]. While a high simulation level is desired, the challenge of scoping the competences to be acquired from the game becomes a challenge. Today, several workplaces aim to empower their workers and therefore push more information to the operators to support a higher degree of decision making. Operators and engineers are becoming knowledge workers [7] and the knowledge intensive workplaces demand more cognitive processes from the operators than merely following procedures.

To address the challenges, we adopted a co-design process using a paper prototype and an iterative design and evaluation process. The paper prototype was designed as a first step in the design of a digital simulation game to support operators in the aluminium plants to increase their understanding of Heat Balance in the electrolysis process. This paper describes the main challenges in designing games in industry and a co-design process using paper and hybrid prototypes to address the challenges. The digital game has been evaluated by several operators and shows potential to support learning and understanding.

The rest of this paper is organised as follows: Section 2 provides a brief overview of games for learning in industry; Section 3 describes the ALTT project; Section 4 describes the challenges; Section 5 describes the game co-design process; Section 6 describes the digital simulation game; Section 7 describes the formative evaluations; Section 8 describes evaluation of learning; Section 9 discusses the results and Section 10 concludes the paper.

\section{Games in Industry}

Serious games have been identified as an effective means for learning a variety of skills and competences in the workplace. Overviews of games that are used in industry to support learning in industrial workplaces are provided in $[6,8]$. A high proportion of the games reported in the literature address the manufacturing and health domains. In the manufacturing domain, several games focus on learning processes and methods such as Lean Thinking [9] or other product development processes, e.g. COSIGA, which was designed to support the education of engineers in the use of Concurrent Engineering for new product development [10]. Most games are designed for learning of hard skills such as product development and manufacturing, supply chain management and logistics (e.g. Beer Game); a review of several games is available from [6]. Risk managements is another area that is supported by serious games; an example BEWARE, is described in [10]. Other industries are exploring the benefits of serious games versus traditional training approaches; e.g. for learning about health and safety in the construction industry [11].

Games have also been designed to support social skills such as communication, collaboration and team working skills; COSIGA is an example where the game enables players to interact through continuous communication and to share and exchange knowledge. Soft skills are often considered more challenging to teach and requires experience to master the skills. The TARGET project, aimed to transfer soft skills related to project and innovation management and sustainable manufacturing using serious games [12]. Learning of soft skills that are expected of an experienced project manager, such as 
stakeholder management, trust building and good leadership was supported through the TARGET game scenarios.

There are several examples of work situation simulators, such as flight simulators and train simulators. These include physical models. Some are available as games, others as professional training facilities. Similarly, chemical/metallurgical processes use simulation models in their operations. However, as far as we know, these simulations have not been used in games for training operators. Games that use physical models, simulating real work situations are rare. In [13], a physical model is used in a serious game to simulate maritime security scenarios in a tactical theatre simulation station. Of course, the accuracy of such models play an important role in the success of the game itself.

Several commercial games and gamified workplaces have been developed in the health sector and other domains to learn procedures and obtain behavioural change. Several such games appear to focus more on the lowest level of the Blooms taxonomy [14], retention and less on the higher levels such as understanding, application and analysis; on supporting the development of cognitive skills. Most games adopt Kolb's Experiential Learning cycle [15] and support reflection at the end of a game session. However, a broader view of reflection such as critical reflection [16] in relation to past and future actions and support for a reflective practice [17] are rarely considered [18].

\section{$3 \quad$ ALTT project}

The ALTT project, aimed at building human capacity in Hydro, is keen to enhance the knowledge of their operators in the aluminium plants so that they will be able to make better and efficient decisions in the workplace. Hydro believes that raising the average competence level of their operators is fundamental to their operations, particularly in their new and automated plant in Karmøy, on the west coast of Norway [19]. Furthermore, they are keen to leverage on the process knowledge that was already available in their simulation models and control systems, which were currently understandable to only the engineers. Thus, the ALTT project brings together the domain expertise and users from Hydro, a game developer, Attensi (www.attensi.com), dynamic process simulation experts, Cybernetica (http://cybernetica.no/) and researchers and designers of Technology Enhanced Learning (TEL) solutions, SINTEF (www.sintef.com).

Aluminium is produced by extracting aluminium from aluminum oxide (bauxite, the raw material found in nature), through an electrolysis process driven by electrical current. Heat Balance is one of the critical process phenomena to understand for the efficient operation of the aluminium production cells, the essence of which involves achieving high productivity of aluminium and low energy loss. This involves balancing energy input against production, by understanding and controlling the complex dependencies among a number of parameters that form the cell, the energy input and how that is varied, and the mass and the composition of the bath.

The focus of the ALTT project is to increase the understanding of Heat Balance Control among the operators and to accelerate the learning process by using simulation games, which leverage on the dynamic process models of the aluminium extraction process. The overall learning goal to be supported by the game is to understand the dependencies among the process variables bath temperature (Tb), superheat ( $\Delta T)$, acidity and side ledge, in Heat Balance in the electrolysis process. Thus, the overall requirement for the game was to support the understanding of how the inputs resistance set point and fluoride additions should be used to control these variables. 


\section{Challenges}

Most industrial environments pose a number of challenges to designing appropriate simulation games and the process industry is no exception. The aluminium production in industry is a complex and challenging domain that is hard to understand for novices. One of the motivations for this project was to support learning and understanding of this complex process and thus, we knew from the beginning that the game should support cognitive processes such as understanding, analyzing situations and applying knowledge [14]. Discussions with the company prior to the project also highlighted that the knowledge among the expert operators was tacit knowledge which they were able to apply and demonstrate but were perhaps not able to explain very well; e.g. the causalities or the consequences of an action [20]. In addition, the learning arena will partly be the workplace rather than a classroom and hence, factors related to the workplace were an important aspect of the design. Thus, the challenges we identified were varied and could be categorized as follows: domain related, cognitive, knowledge dimensions, workplace related as well as the more general game and learning design related challenges. The following paragraphs describe these challenges.

Domain related challenges - the aluminium production cells present a chemically harsh environment with temperatures around $1000^{\circ} \mathrm{C}$, limiting the possibility to use sensors to provide continuous or real-time values of the key process variables within the cell, such as the temperature or the chemical composition of the bath mass. Therefore, the process values within the cells have to be calculated or simulated based on their inputs and outputs. Thus, the amount of measurable values is limited and new measurements are available typically once every 18-36 hours. By combining the process knowledge that is already available in the simulation models, these measured values along with inputs can be used to predict process variable values some hours into the future, every 5 minutes.

Cognitive challenges - parts of the chemical process is slow, often taking up to three weeks before the consequences of an action on the cell is seen; e.g. the addition of fluoride. Process values within the cells are in general not available, and control actions are taken mainly based on the limited measurement information that is available, typically every 1836 hours. For the operators, this makes it hard to understand what happens between the measurements. This separation of action and causality makes it harder for the operators to envisage the consequences of their actions and to build an understanding of the dependencies among the parameters that determine the status of the cell [17]; e.g. how the addition of fluoride affects the composition of the bath mass and the temperature in the cell. The actions that are conducted on the cell require a cognitive decision making process that takes into account a huge number of parameters and their complex dependencies. Before determining a course of action, the operators must first establish the status of the cell and how it got there, by assessing the behavior of the cell over the past three weeks or so. Then, they must anticipate the consequences of the possible action or actions available to them, before selecting their actions.

Knowledge dimension related challenges - Hydro, like many industrial workplaces, have detailed and well-defined Standard Operation Procedures (SOP) including procedures to handle known and common deviations. As industry moves towards a more knowledge intensive one, the challenge often lies in acquiring non-procedural knowledge that takes into account the complexity of the domain and requires a good understanding of the domain. Today, operators master the complexity of Heat Balance Control through an apprenticeship program [21], which includes following and working with experienced operators and often takes several years. The experienced operators, referred to as "cell doctors" by their colleagues, have mastered the complexity of Heat Balance Control through experience and has a lot of tacit knowledge. It is difficult for them to externalize their tacit knowledge. Thus, novices often learn patterns from the experts rather than acquire a deep understanding of the process and why things are done the way they are. This is sometimes a challenge, particularly if the cell technology changes, as procedures and old habits that were 
appropriate for one technology may not be appropriate in the new setting. The operators thus require a deep understanding to know what has changed and why to make the appropriate decisions.

Workplace related challenges - one of the main challenges in designing games for industry is finding the right level of conceptualization and abstraction while keeping it close to the real workplace situations; i.e. determining the appropriate simulation level [6]. In the case of ALTT, a very high level of fidelity of the domain was available through the simulations of the dynamic process. However, incorporating these models and interfaces in an engaging game that supported understanding the complexity of the domain and stimulated the desired cognitive processes among the operators is a challenge.

Game and learning design related challenges - while a game with a high level of fidelity and very close to the operations in the aluminium production plants was desired, this posed challenges in designing the learning support and ensuring that operators with different levels of expertise would benefit from the game. The game simulated the workplace and learning at the workplace as a part of the daily work routines, which often required a good understanding of many things such as the complex domain, SOP and a reflective practice. Thus, breaking down the overall learning goal into smaller ones and the knowledge or the learning content (available through the dynamic process models) into simpler ones are challenges in designing the learning path. This also required determining the scope of the learning contents (skills level as described in [6]) and identifying the appropriate game mechanics for the relevant learning mechanics [22].

\section{Game Co-design process}

We adopted a co-design and iterative game design and development process, as illustrated in Figure 1, to address the challenges posed by the domain. A co-design approach ensured adequate interaction with the end users and seemed an effective way to tap into the tacit knowledge of the experts. This approach enabled better access to the collective needs, knowledge and creativity of diverse user groups such as the novices and experienced operators. Furthermore, as the designers and developers of the game were novices as well, the co-design process provided an opportunity to obtain a better insight into the needs of the operators, which complemented other approaches such as observations [23].

The process began with requirements elicitation workshops with novice and expert operators (the end users) and domain experts. A paper prototype was then developed to validate the concept for the game design and to identify the relevant game mechanics. This was followed by a hybrid prototype, which combined both digital and non-digital capabilities. Finally, a completely digital prototype was developed.

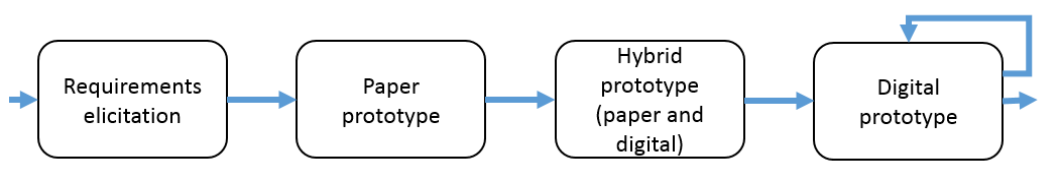

Figure 1. Co-design and iterative game development

In addition to addressing the challenges described earlier, and given the ambition of the project to bring learning close to the workplace, an iterative co-design process at the initial stages of the design was essential for two important reasons: i) to ensure that the expectations of the different user groups were met sufficiently to increase the uptake of the game, and ii) to motivate and engage the users and cultivate a sense of ownership towards the game. Engaging the operators as partners in design was thus an important part of the process. The aluminium production plants were located in different parts of Norway and had different production technologies. Thus, co-design workshops were conducted in more than one plant to ensure input from the different plants. 
The overall design process consisted of iterations of development, validation of concepts and prototypes with operators and experts from Hydro and improving the design based on the feedback. During the iterations of the development, other user-centred methods were used such as usability testing. These iterations were a part of the design and formative evaluations of the final game that was developed.

\subsection{Requirement Elicitation}

The requirements elicitation workshops were designed to focus mostly on the cognitive, knowledge related and game design related challenges. The workshops were conducted as focus group discussions with different user groups - the operators, action responsible operator and production and overall process management staff. Separate discussions were held with each group to ensure that each group could speak freely; in particular to ensure that the less experienced operators felt confident in expressing their views without the presence of their superiors. In addition, a common discussion was held where all the user groups were included.

The discussions were inspired by Cognitive Task Analysis methods [24], [25]; how operators make decisions, the problems they face, what they found most challenging and why, and the cues and strategies that they use [26]. They helped us not only to obtain a good insight into the cognitive challenges faced by operators but also to identify expectations from the game in meeting the needs of the workplace. More importantly, this was also a significant step in seeding the idea of a new type of learning environment and starting to engage the end users right from the start.

\subsection{The Paper Prototype}

The aims of starting with a paper prototype was to identify how best to address the cognitive and workplace related challenges. In addition, paper prototyping is common among many games and applications development fields to avoid using resources in coding before the concepts were validated.

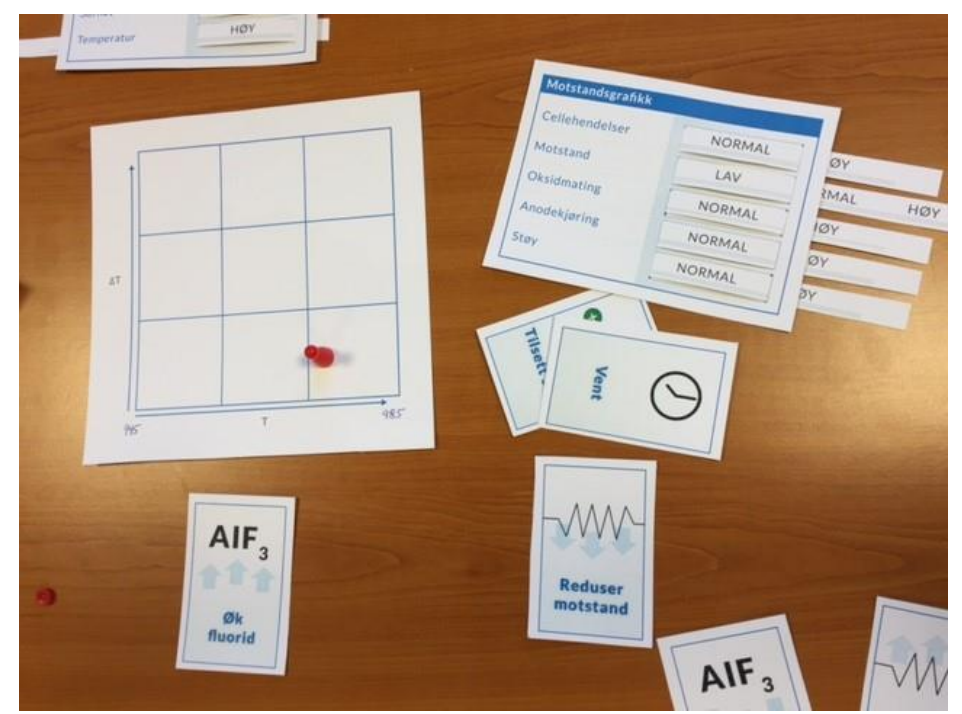

Figure 2. ALTT Heat Balance game - Paper Prototype

The starting point for our design was the domain knowledge of the Hydro experts in the project, the teaching material that was already used at Hydro for the theoretical part of the training of operators and the input from the requirements elicitation workshops. Two of the non-Hydro participants of the ALTT project had previously attended a theory course on Heat Balance Control and used this material. One of the key abstractions that were used in the course material was a 9-cell matrix to illustrate the dependencies among two pf the 
parameter values (the bath temperature and the superheat) and how they varied as other parameters were varied. We leveraged on this abstraction and designed the game concept and game mechanics around this. This was developed as a paper prototype, shown in Figure 2.

The paper prototype was low fidelity as the focus was on capturing and validating the relevant concepts and the conceptual correctness of the prototype. The game is designed around the 9-cell matrix, shown on the top left of Figure 2. This is the essence of the conceptual model that captures the interdependencies of the parameters, bath temperature and superheat, associated to the electrolysis process. The current state of a production cell is shown as a dot position in the 9-cell matrix. It was a turn-based games and the game is played by selecting one or more actions that will be taken on a cell. The action cards are shown to the bottom right of the figure. The actions available in the game are changing the resistance and the amount of fluoride (acidity) in the cell, wait without taking any action or add soda to the cell. Once an action is taken, the 9-cell matrix is updated with the new state of the cell. The consequences of the actions, which were the new values of some of the parameters of the dynamic process, are also shown by sliders, shown to the top right of the figure. The sliders are used to indicate if the player's actions caused an increase, decrease or had no effect on the parameters.

The paper prototype was presented to several novices, experienced operators and domain experts, who provided their feedback. In addition, two novices and two experienced operators tried the paper prototype, with a game master that determined the next state of the game, based on the player's actions in the game. These sessions with the operators also provided a useful discussion arena and valuable input for refining the game concepts.

The paper prototype proved to be a very useful artefact for the co-design process. It helped to identify things we didn't foresee at the beginning, clarification of the scope and the appropriate level of granularity (e.g. of graphs) and that we should show continuous states rather than discrete states of the cell; i.e. the need for a model to show that it is a dynamic process. It was also instrumental to access operators' tacit knowledge and stimulated interactions with the different stakeholders.

The most important feedback we received from the users was that the concept was good and would be very helpful in learning about Heat Balance Control. Thus, we decided to go ahead with the 9-cell matrix and the action cards. However, the operators wanted the game to be high fidelity and resemble their daily workplace applications. This meant that rather than seeing the consequence of their actions as a relative change (i.e. normal, high and low), they wanted to see the real change, as accurate as possible. They also indicated the specific parameters that they used in their daily decision making and thus asked to include those parameters in the game. Workshops with the operators using the paper prototype played an important role in clarifying the scope of the game. Discussions during the workshop also included determining the win-state for the game and the number of rounds that a player would be able to play before losing the game.

\subsection{Hybrid prototype}

The feedback from the workshop using the paper prototype highlighted the challenges in designing the game to meet the specific requirements for the operators' workplace. Based on the feedback, the game design was enhanced to connect the game directly to the simulation of the process model, which would be a significant part of the learning content. This led to a high fidelity hybrid prototype, where the 9-cell matrix and action cards from the paper prototype were used along with a digitized version of the consequences of the player's actions. A Microsoft Excel based visualization of the consequences were simulated real time, using the underlying simulation model of the dynamic process. A photo from the workshop using the hybrid version of the game is shown in Figure 3, where the display on the screen shows the digital parts of the hybrid prototype. The hybrid prototype was evaluated by four novice operators and two domain experts. The players used the action cards from the paper prototype. Instead of the sliders from the paper prototype, the graphs 
pag. 46

to the left of the screen display show the values on graphs and the player is able to see how the values have evolved during the course of the play. To the right of the screen, the values of the parameters are shown on the game board, the 9-cell matrix.

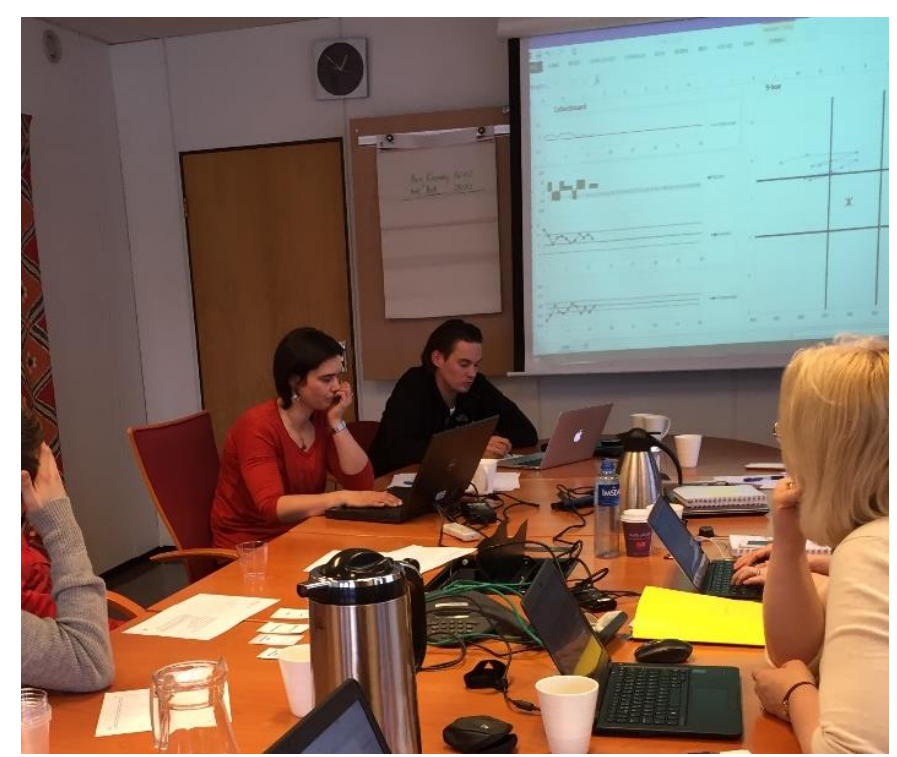

Figure 3. ALTT Heat Balance game - Hybrid Prototype

The hybrid prototype helped to increase the trust and enthusiasm among the operators. Valuable feedback was received in fine tuning the level of granularity and the knowledge contents that should be included in the game and the user interface. One of the most important feedback was related to the cognitive challenge of a reflective practice and supporting the necessary cognitive processes before selecting an action; i.e. looking at the history of the cell, as this would have implications on the choice of the current action. This was very valuable to identify detailed requirements on how the game should be implemented, it's interaction with the simulation model and the choice of the game scenarios.

\section{ALTT Simulation Game}

A digital game was developed based on the requirements from the users and the feedback received from the workshops with the paper prototype and the hybrid prototype. Since the electrolysis process is a dynamic process, one of the most important design decisions to meet the domain related challenge was to implement a dynamic process model of the cell that could simulate future states of the cell. The dynamic process model is based on a simulator model developed and used by Hydro over several years. Thus, this was a wellproved model for describing the main dynamics of the Heat Balance in an electrolysis cell. In order to work as a process data provider for the game, minor modifications needed to be made and some new interfaces needed to be implemented. In the game, the dynamic process model starts from a set of process parameter values defined by the game scenario. The simulation model is coded as a separate simulator behind the scenes of the game. When a player selects a game scenario to play, the simulator is initialized with parameters and process variable values from a set of predefined conditions related to the specific scenario. For each turn in the game, the chosen actions from the player are sent to the simulator which calculates the process behavior in the next time period. The new process state is then returned to the game and the player presented with the result of her/his actions, and the game continues. The game and simulator interaction is illustrated in Figure 4. 


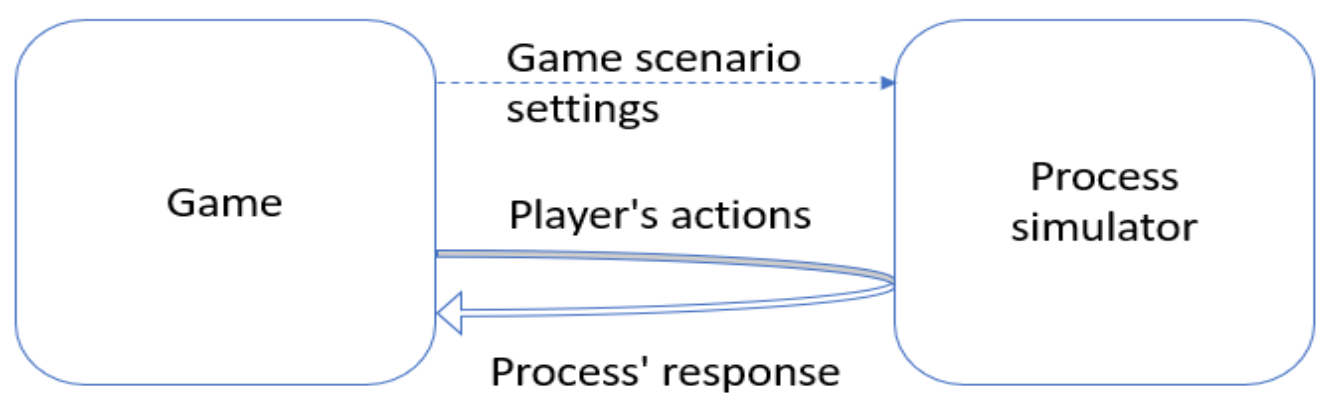

Figure 4. Interaction between game and process simulator.

The main screen for the ALTT digital prototype is shown in Figure 5.

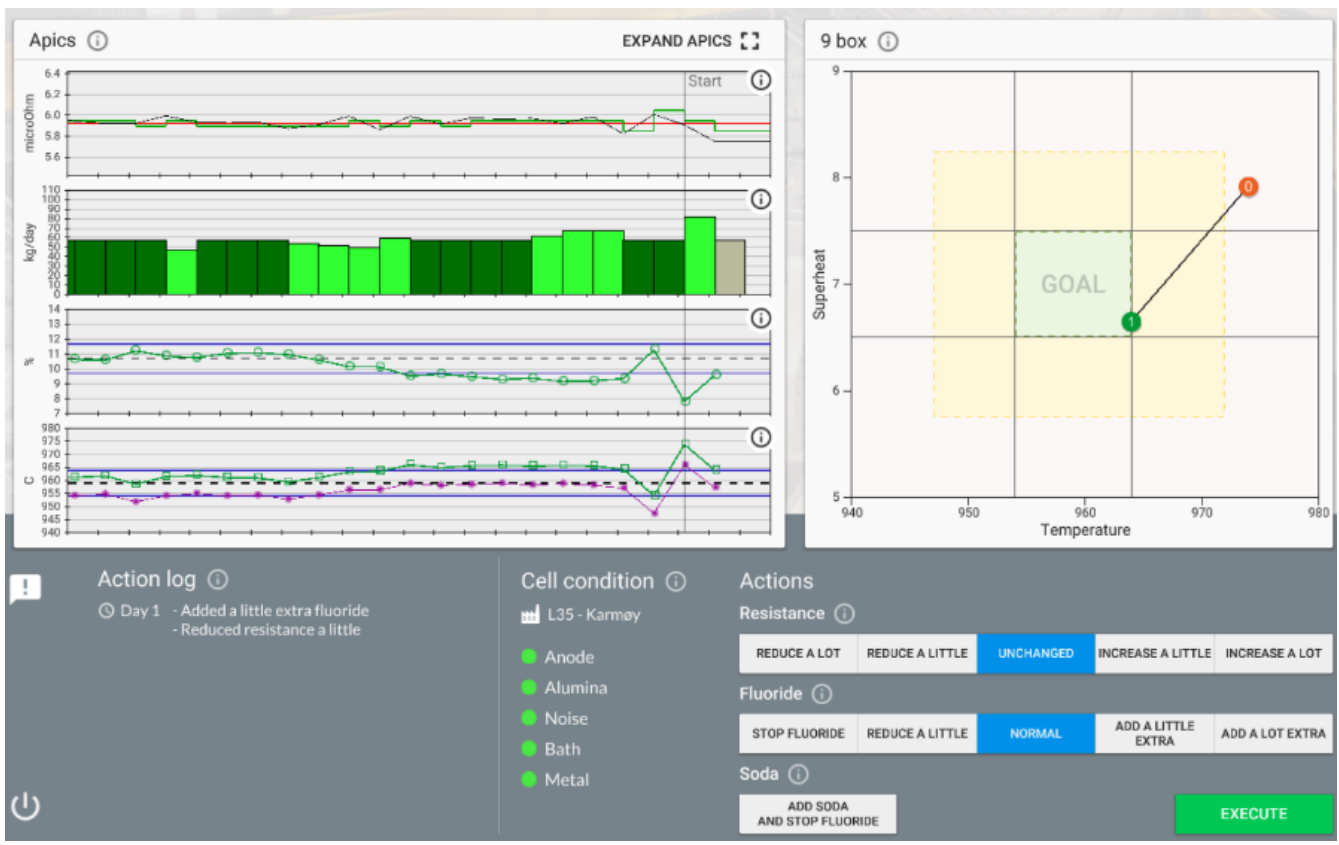

Figure 5. ALTT Heat Balance game - Digital Prototype

The 9-cell matrix is to the top right of the screen and the action buttons are to the bottom right. The values of the axes of the 9-cell matrix indicate the range of values that are within normal or acceptable deviations. The values for middle cell for the middle cell indicate the normal state of the cell. Thus, the win-state for the game is when a player manages to stay within the middle cell of the 9-cell matrix for three consecutive turns, where each turn is 20 or 24 hours; i.e. the player is able to bring a cell to normal state and maintain it in the normal state. The time period between each turn is defined by the frequency of the main measurements or operations that are performed in the workplace, which depends on process practice and can vary from plant to plant and is typically between 18-36 hours. The player loses after 20 turns without winning or when the player takes an action that results in parameter values that are outside of the range of the 9-cell matrix, which indicates that the player has managed to get the cell into an extreme deviation state.

The other main GUI component is the cell's historical information, which is shown as a set of graphs, which include information on resistance, fluoride additions, acidity and bath temperature. Additional information the operators use in their decision making process, which indicate the current state of the cell, are shown in the middle in the bottom part of the screen. To simplify the context, it was decided that the concept of traffic lights will be used; and these values are shown as normal, using the green indicators. A history of the player's actions during the game session are shown to the bottom left of the screen. 
pag. 48

The digital prototype facilitated discussions around the learning design such as how the scoring could be done, how the scores could be communicated to the users and how the learning support, such as hints and help should be designed for the different expertise levels of the users.

The digital game has been further enhanced with capabilities to support reflection after a game session and particularly while playing the game. To ensure that players reflect upon their actions while playing the game, functionality has been added for players to anticipate the consequences of their actions. Three questions presented to the player after each action is designed to stimulate them to anticipate the consequences of their actions; see Figure 6. Feedback on the reflection is provided immediately by showing the player's anticipated consequences and the actual consequences (simulated using the dynamic process model) as shown in Figure 7.
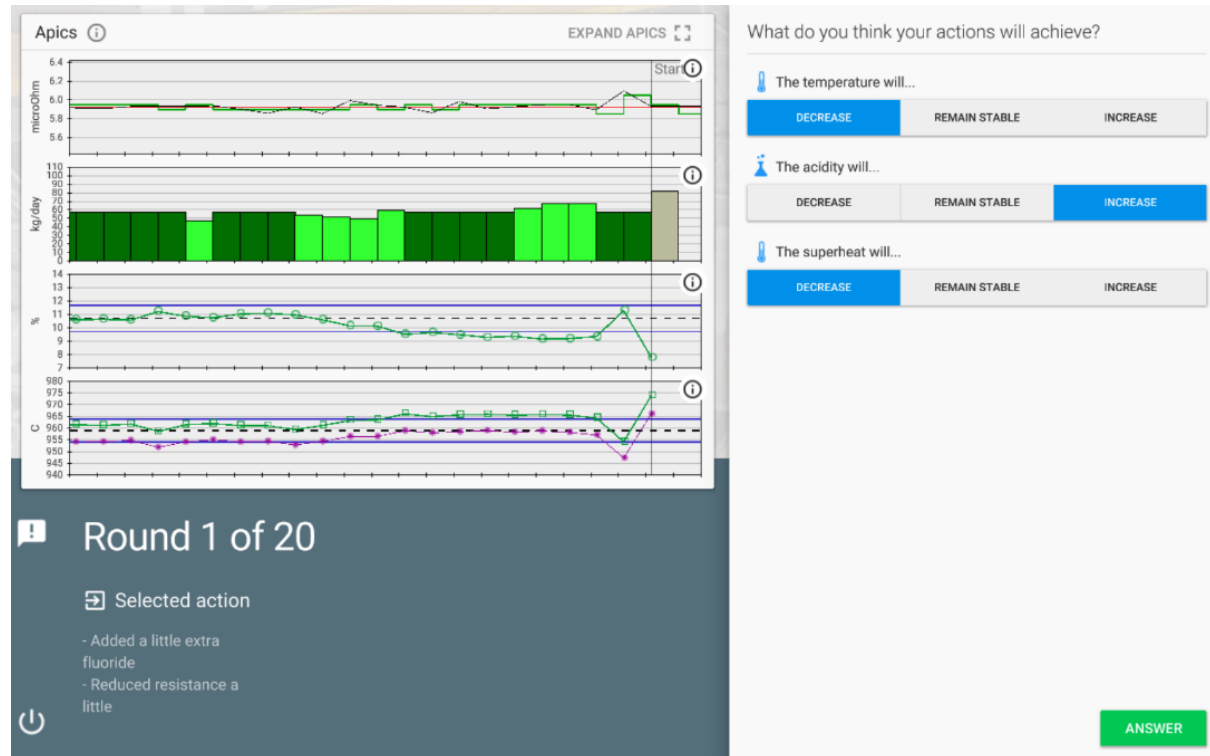

Figure 6. ALTT Heat Balance Game - In-game Reflection Trigger
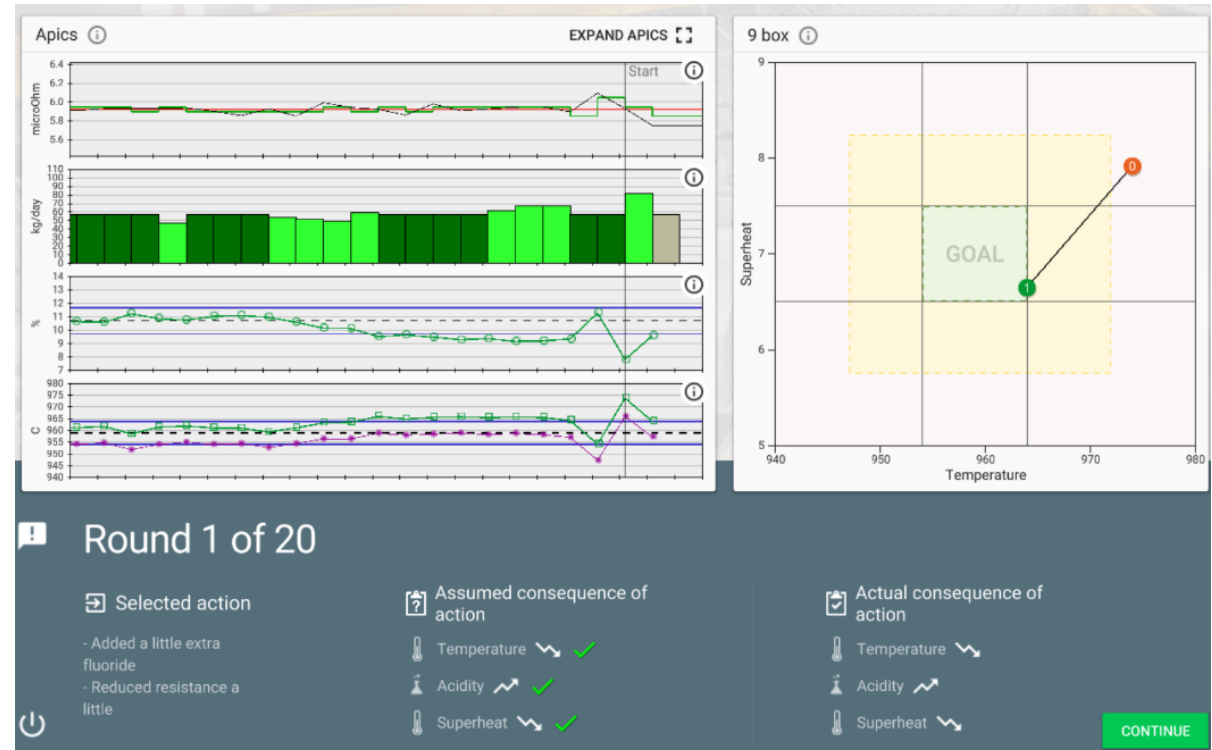

Figure 7. ALTT Heat Balance Game - In-game Feedback on Reflection

The support for reflection is designed based on the Reflection Continuum Model, described in [27], and reflection is described as examining the current status, looking at the history shown in the graphs and looking ahead by anticipating the consequences of their actions. 
Additional concepts relevant for the domain have been supported explicitly such as the heat loss from the cell and how that would relate to the side ledge of the cell and to the parameters shown in the digital prototype in Figure 5, the bath temperature, superheat, resistance and acidity. The additional concepts and complexity built into the game also required additional GUI elements, such as a simple visualisation of the side ledge. Thus, functionality to define the specific GUI elements for specific scenarios and learning goals is currently in place.

\section{Formative Evaluation}

As described in Section 5, an iterative co-design and evaluation process was followed. To ensure feedback from as many operators as possible, several formative evaluations were conducted with the digital prototype, at Hydro's four aluminium production plants before the final version of the first prototype of the digital game was released. In addition to the general improvement of the game, the formative evaluations had two main objectives:

1. Validation of the dynamic process model to ensure that the aluminium production cell that is simulated in the game behaved correctly.

2. The graphical user interface and game play.

The formative evaluations focused on the incremental developments of the dynamic process model and the functionalities of the game. Thus, each evaluation focused on the refined and new functionalities since the previous evaluations.

\subsection{Validation of the Dynamic Process Model}

The validation of the dynamic process model was essential to the success of the game, its usability and to ensure that the players learnt the correct knowledge contents. The dynamic process model has been developed and evolved over several years. Thus, the model has been through several rounds of technical process validation with process data prior to being used in the game. However, any model will have limitations and can only describe certain aspects of reality in a precise way. The phenomena described in the game was, to some extent, formed in a way that the model could support, while less important phenomena that were not supported by the model were omitted in the game. Then, the behavioral aspects of the model, as experienced by the operators was validated by two expert groups, the process experts from Hydro and the company that develops these models, Cybernetica, and experienced operators from Hydro. The first group consisted of project participants. The second group of experts participated in formative evaluation workshops, where they played the game and provided feedback on the model and the game. Both the expert groups were able to see how a cell evolved through a game session and how the cell responded to their actions in the game and they were able to detect when the cell did not behave as expected. Based on the feedback from the experts, the dynamic process model was refined until it satisfied the experts.

\subsection{Graphical User Interface and Game Play}

The main methods used for the evaluation of the GUI and the game during the formative evaluations were observation of players followed by a focus group discussion and/or a semi-structured interview. Each formative evaluation had a specific focus so that it was easier to determine where the focus of the observation would be and to gather data. After each evaluation round, the project group discussed and agreed upon the important requirements that were gathered from the evaluations and how they would be prioritized.

The game was installed on PCs, which were set up around a table. Four game scenarios were provided where the level of difficulty of the scenarios varied from easy to difficult. Novice operators and experienced operators participated during the formative evaluations. 
The players were asked to play for around 30 minutes, either individually or in pairs. When they played in pairs, the discussions between the two players in each pair were taken into account, to understand if and what they may find difficult and other design related issues. The data was gathered as notes, which were then processed as requirements to refine the GUI and the game design.

The formative evaluations provided valuable feedback on the scope of the information that should be provided in the game, the granularity of it and on the GUI and usability. The need for additional learning support such as explanations of concepts, hints, help and feedback were identified and designed based on the input from these workshops.

\section{$8 \quad$ Evaluation of Learning}

Summative evaluations were also conducted in the different Hydro aluminium production plants in Norway. The overall evaluation method was inspired by Mayor et al.'s comprehensive conceptual framework to support the design, data gathering and evaluation of serious games [28]. Mayor et al.'s framework takes into account the pre- and post-game conditions such as the learners' knowledge and attitudes; mediating variables such as learning styles and the context of learning. The following sub-sections describe in detail our evaluation methods and the results.

\subsection{Evaluation Method}

The evaluation method we used was based on self-reporting pre- and post-intervention questionnaires and knowledge tests, combined with in-game session log data [28]. The main focus of the evaluation of learning was the increase in knowledge about Heat Balance by playing the game. The knowledge questionnaires were designed to evaluate understanding and the application of knowledge rather than factual or procedural knowledge. Knowledge tests were conducted as pre- and post-intervention knowledge questions related to the contents in the game scenarios. In addition, changes in the learners' attitudes towards learning Heat Balance Control, learning using games, perceived learning and confidence were taken into account. The pre-intervention background questionnaire included information on demographics, learners' experience and use of technology and games. In addition to the information related to learners' attitudes, the post-intervention questionnaire included also information related to usability, transferability of the game to the workplace and specific capabilities in the game. The session logs from the game were designed to support the analysis of the learners' behavior; e.g. reflection during a game session, when taking an action in the game.

We have conducted four summative evaluations with operators. Two of the evaluations (8 and 28 participants) were based on pre- and post-intervention evaluations, where the participants were provided access to the game, to play in their own time; see Figure 8 . The participants were operators with varying levels of experience. They were provided access to the game for 2 weeks, where the game installed on a few computers in a common area in their workplace. The players were asked to play the game in their own time.
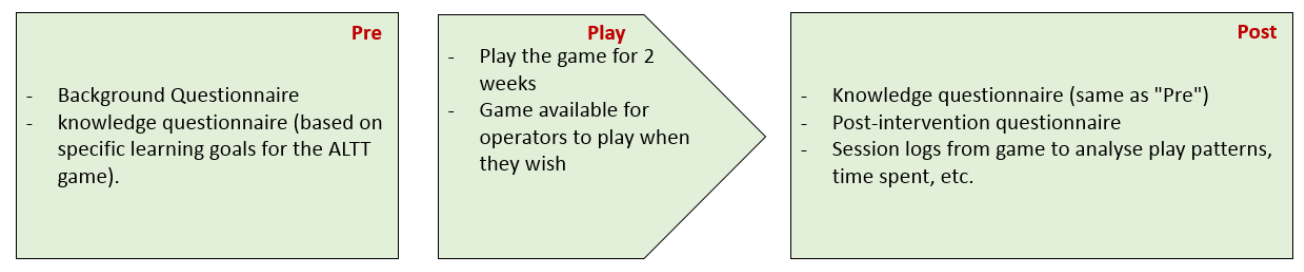

Figure 8. Evaluation Method - game as a standalone application 
The other two evaluations (12 and 16 participants) were conducted, where the game was used as a part of a classroom course to support reflection through discussions. In these two studies, participants were asked to play the game in pairs and talk aloud. In both these cases, the instructor was able to observe and interact with the participants during the course. The instructor introduced some theory and a scenario followed by a play session, where the players were able to play the relevant game scenario(s) and discuss with their partner. This was in iterative process as shown in Figure 9. Similar pre- and post-intervention and knowledge questionnaires were used. An additional post-intervention questionnaire was used with a few questions that addressed the specific goals of the study related to the role of the game as a part of the course.
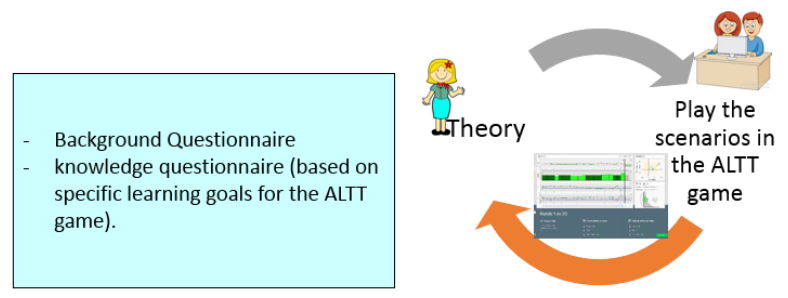

Knowledge questionnaire (same as "Pre") Post-intervention questionnaire (general) Post-intervention questionnaire (specific for the goals of the study) time spent.

Figure 9. Evaluation Method - game as a part of a course

\subsection{Evaluation Results}

The different evaluation studies were conducted over a period of one and a half years, where the game interface and the formulation of the questions were refined. Hence, we have chosen to report the results from the knowledge questionnaires separately. Nevertheless, the results from the knowledge questionnaires, from all four evaluations, show that there was knowledge gain for $80 \%$ of the participants, who completed both pre- and postintervention knowledge questionnaires. The mean knowledge increase was $61 \%$. The results of the knowledge questionnaires for two of the studies are shown in Figure 10 and Figure 11. The vertical axes show the no. of correct answers and horizontal axes show the participants. In Figure 11, A are the least experienced operators and $\mathrm{C}$ are the more experienced operators.

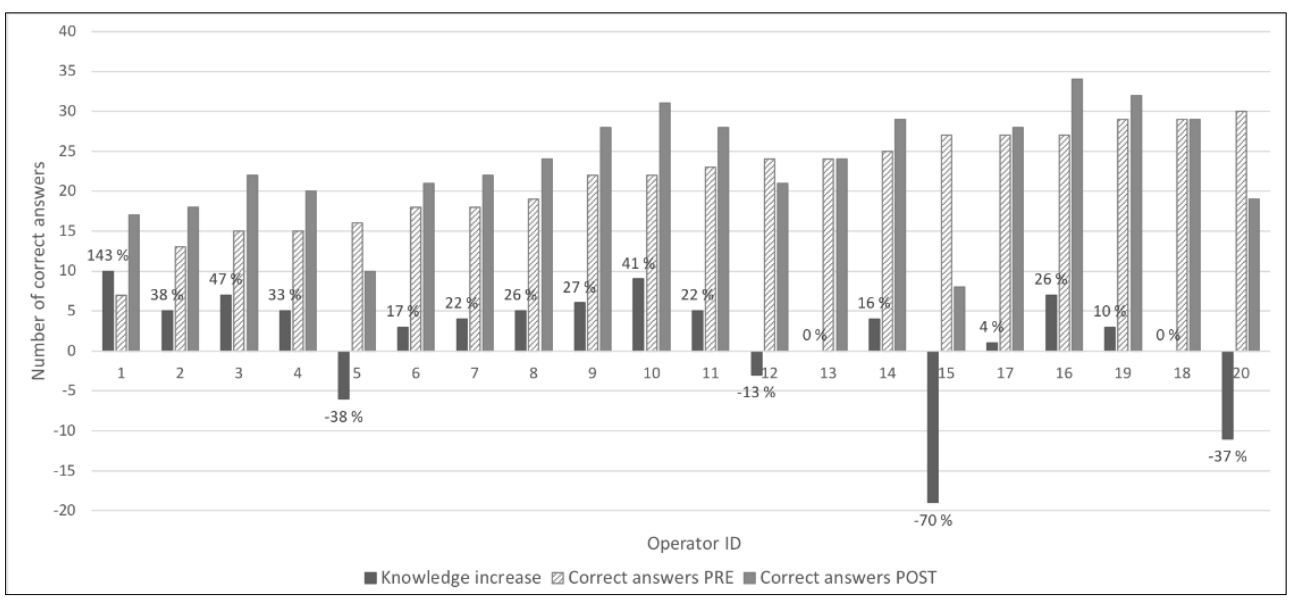

Figure 10. Evaluation of learning - Operators play in their own time 


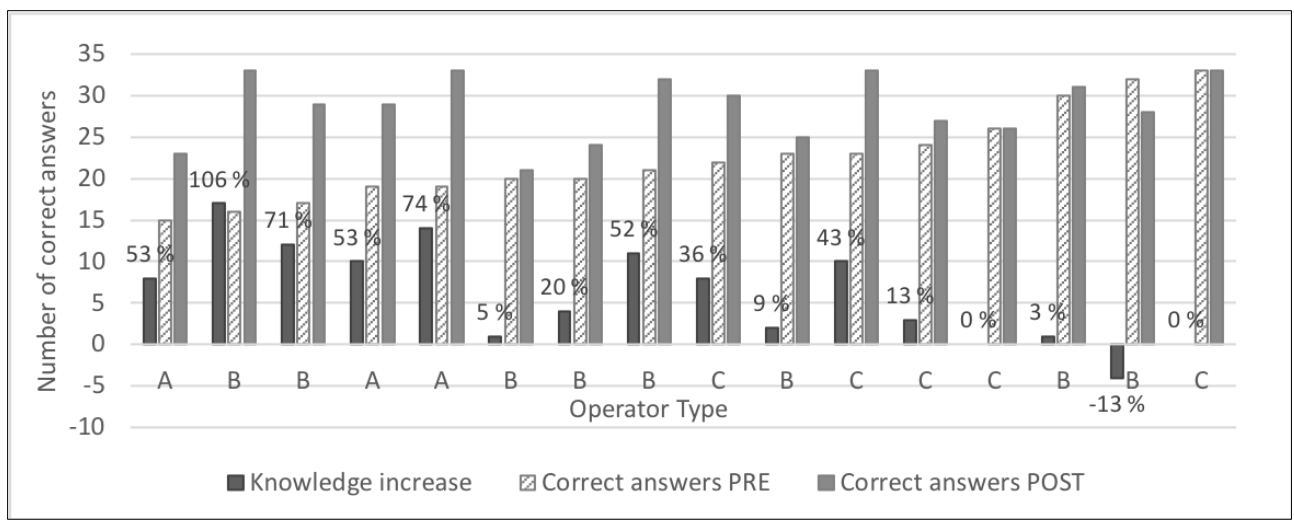

Figure 11. Evaluation of learning - game as a part of the course

The results show that the game has the potential to support learning for operators with different levels of experience. One of the most important conclusions that we could make from the results is that in general, the operators with less prior knowledge gained more from playing the game. We can see that knowledge gain is higher among the operators that had less previous knowledge than the ones that had a higher level of knowledge from before. Note that in Figure 10, the high negative values on the knowledge gain is due to blank or partly blank post-knowledge questionnaires that were submitted by some participants. A possible explanation for this could be that they felt they understood everything and therefore did not see any reason to complete the knowledge questionnaire a second time.

In addition to the knowledge questionnaires, we also analysed the session logs from the game to evaluate if the learners actually understood the consequences of their actions in the game, i.e. the consequences of increasing/decreasing the addition of fluoride and resistance; see Figure 5. After each action in the game, three questions were presented to the player to stimulate reflection and anticipation of the consequences of their actions on the three process variables bath temperature, acidity and superheat (which is a non-measured, abstract concept which is important for Heat Balance); see Figure 6 and Figure 7. The three graphs in Figure 12 show the average response to the three questions from one of the studies where the players were given access to the game to play in their own time (28 players and across 8 game scenarios shown on the horizontal axis). The green bars (at the bottom of the graph) show the correct answers; the brown bars (on the top of the graph) show the incorrect answers and the white bars (in the middle) show an error by one. It can be seen that there is a higher percent of correct answers than incorrect ones. Since the \%age of correct answers is higher than 33\%, it can be assumed that players were not randomly selecting an answer, but making a conscious choice of the correct answer. Players seem to understand the concept of temperature better than acidity and superheat. The session logs from the other three studies also confirm this result.
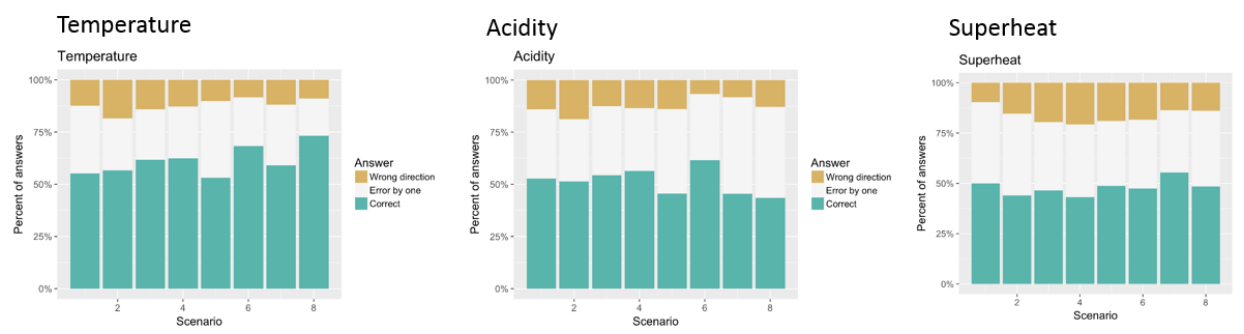

Figure 12. Evaluation of Learning - Understanding of concepts 


\section{Results and Discussions}

The results from the knowledge tests and the evaluation of the session logs from the game suggest that the game has the potential to support learning and understanding of the concepts and dependencies that are important in Heat Balance Control. The results shown in Figure 10 and Figure 11 indicate that the game can support learning for operators of different competence and experience levels. During discussions with the more experienced operators, they highlighted the benefit of playing the game to refresh their competences and to explore uncommon situations. The expert operators, who often act as mentors to the novices, saw the game as an important medium to explain situations and stimulate discussions among the operators. These are indications that the design of the game and the game scenarios address the challenges that were described in Section 4.

We have also analyzed the pre- and post-intervention questionnaires to address specific aspects that address the challenges described earlier. The main domain related challenge related to the instrumentation of the aluminium cell and making the process variables and changes to them visible and explicit to the operators. The design decision to embed the dynamic process model in the game to determine the next state was significant in addressing this challenge. The most common feedback from the operators was that through the game, they could see the consequences of their actions immediately without having to wait for several days, sometimes weeks, as they would in real life. Similarly, using the abstraction of the central concepts (bath temperature, acidity, resistance and superheat), the 9-cell matrix as the game board proved to be a simple, yet effective means of showing the complex dependencies among the parameters. Over 70\% percent of the participants from the studies strongly agreed to the post-intervention question "The game board (9-cell matrix) provided feedback on if I was on the right path to achieve the goal".

The dynamic process model also proved to be an essential part of addressing the cognitive challenges, in particular, supporting the understanding of causality of the actions taken by the operators in their workplace. For the post-intervention question "The questions I got about temperature, acidity and superheat helped to think about the consequences of my actions", 38\% of the participants strongly agreed and 30\% agreed. In addition, the model and the reflection questions to stimulate reflection-in-action [17] during the game seem to indicate that the players indeed had an understanding and were most likely making conscious and intentional decisions about their actions in the game, see Figure 7. These questions helped to make explicit the important parameters and how each one of them impacted the cell and how it evolved. This was also important in addressing the knowledge related challenges and contributed towards externalizing some of the tacit knowledge of the experts and present them in a manner that less experienced operators could understand.

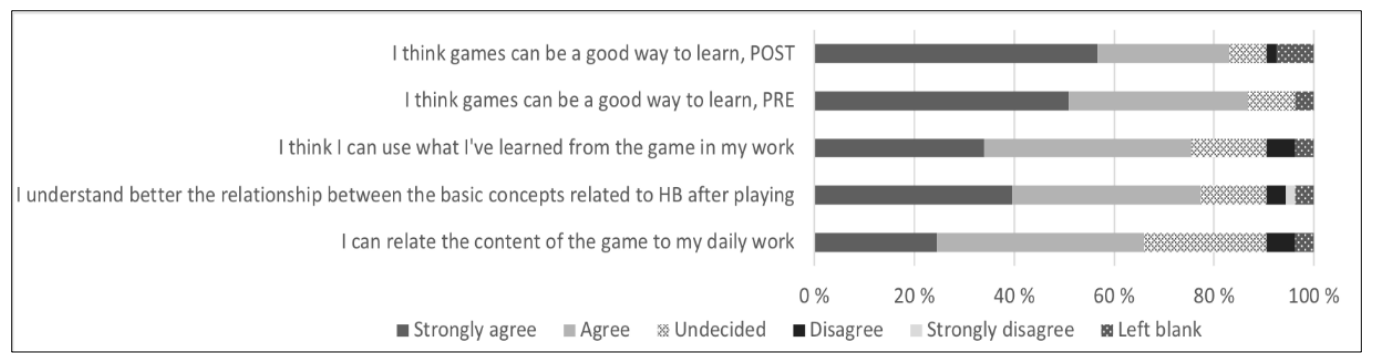

Figure 13. Evaluation of the Game - Attitudes, Usability and Transferability

Some of the indicators for the workplace related challenges are captured in the postintervention questions shown in Figure 13. Players' perceptions of usability of the game in their work and if the contents of the game could be related to their work are indicators of how well a game addresses the workplace challenges. These are indeed important factors for the operators to use it as a part of their daily work and to help them understand why they do things in certain ways. The responses to the post-intervention questions were positive to 
pag. 54

two questions that address usability (I think I can use what I've learned from the game in my work) and transferability (I can relate the contents of the game to my daily work). In addition, attitudes towards learning using games and players' own perceptions of learning from playing the game are positive, which can be considered as indicators of appropriate game and learning design.

\section{Conclusions}

This paper described the digital simulation game designed and developed for the operators in aluminium plants to master the cognitive skills required for Heat Balance Control in the aluminium production cells. Designing games for learning in the workplace is challenging in many ways. The aluminium productions cells are harsh environments that are difficult to instrument and pose domain related challenges to represent the workplace appropriately in the game. Several cognitive challenges arise due to the slow nature of the physical process, disconnecting the operators' actions and causality. In addition, the complex chemical process demands a high level of understanding on the parameters and their complex dependencies. The experience of the experts remains as tacit knowledge, often unavailable to the operators. Furthermore, additional challenges are related to representing an appropriate level of detail and precision of the workplace.

A co-design process, using a paper prototype and a hybrid prototype of the game, and iterative development and evaluation cycles are described. The digital simulation game is described in detail. One of the main design decisions that helped to address the challenges was the use of a high fidelity dynamic process model to determine the new states of the cells during the game play. The evaluation results show that the game has the potential to support learning and appears to meet the expectations of the operators. The co-design process, several discussions and evaluation studies with the different user groups and stakeholders were invaluable in the development and evaluation of a game concept that meets the expectations of the users.

The work has been conducted within the Norwegian research project, ALTT, for the aluminium producer Hydro. We are currently planning to use the game as a part of the formal apprenticeship training process with their newly employed trainees. In addition, we are also discussing with the company on how to incorporate the game as a part of their competence development program.

\section{Acknowledgement}

This work has been conducted within the ALTT project funded by the BIA program, Norwegian Research Council. The authors would like to thank the participants of the workshops and evaluation studies, the operators and the experts from Hydro who have provided valuable feedback through discussions and evaluations, Torstein Rotevatn from Cybernetica for his contributions in developing the dynamic process model, Andreas Landmark, Hans Torvatn and Anette Østbø Sørensen, from SINTEF, for their contributions with the questionnaire design and data analyses.

\section{References}

[1] S.A. Petersen and P. Hold $\varnothing$, Dataspill hjelper industrien, in Dagensnaeringsliv. 2017: Norway.

[2] D. Pratt and J. Johnson, The Apprenticeship Perspective: Modelling Ways of Being, in Five Perspectives on Teaching in Adult and Higher Education, D. Pratt, Editor. 1998, Krieger Publishing Company: Malabar FL.

[3] C.N. Quinn, Engaging learning. Designing E-Learning Simulation Games. 2005, San Francisco. : Pfeiffer, John Wiley and Sons, Inc. 
[4] F. Bellotti, R. Berta, A. De Gloria, M. Ott, S. Arnab, S. De Freitas, and K. Kiili. Designing Serious Games for education: from Pedagogical principles to Game Mechanisms. in 5th European Conference on Game-Based Learning. 2011. Athens, Greece: Academic Publ. Ltd, Reading, UK.

[5] K. Killi, Digital game-based learning: Towards an experiential gaming model. The Internet and Higher Education, 2005. 8(1): p. 13-24. https://doi.org/10.1016/j.iheduc.2004.12.001

[6] J.C.K.H. Riedel and J.B. Hauge. State of the Art of Serious Games for Business and Industry. in 17th International Conference on Concurrent Enterprising (ICE 2011). 2011.

[7] Global education in manufacturing strategy. Journal of Intelligent Manufacturing, 2011. 22(5): p. 663-674. https://doi.org/10.1007/s10845-009-0326-2

[8] H. Duin, J.B. Hauge, F. Hunecker, and K.-D. Thoben, Application of Serious Games in Industrial Contexts, in Business, Technological, and Social Dimensions of Computer Games: Multidisciplinary Developments, M. Cruz-Cunha, V. Varvalho, and P. Tavares, Editors. 2011, Information Science Reference: Hershey, PA. p. 331-347. https://doi.org/10.4018/978-160960-567-4.ch020

[9] B. Pourabdollahiana, M. Taisch, and E. Kergaa. Serious Games in Manufacturing Education: Evaluation of Learners' Egagement. in Virtual Worlds for Serious Applications (VSGAMES'12). 2012. Elsevier B.V.

[10] J.B. Hauge and J.C.K.H. Riedel. Evaluation of simulation games for teaching engineering and manufacturing in Evaluation of simulation games for teaching engineering and manufacturing 2012. https://doi.org/10.1016/j.procs.2012.10.073

[11] Y. Gao, G.V. A., and Y.T. W., Serious Games vs. Traditional tools in Construction Safety Training: A Review., in Joint Conference on Computing in Construction (JC3). 2017: Heraklion, Greece. https://doi.org/10.24928/JC3-2017/0070

[12] M. Oliveira, B. Andersen, and H. Torvatn, Rapid Competence Development, in Advances in Technology Enhanced Learning, F. Wild, P. Lefrere, and P. Scott, Editors. 2013, Open University Press.

[13] R.T. Kessela and A. Tesei. Accuracy Requirements for Physical Models in Serious Games. in Virtual Worlds for Serious Applications (VS-GAMES'12). 2012. https://doi.org/10.1016/j.procs.2012.10.095

[14] L.W. Anderson and D.R. Krathwohl, eds. A taxonomy for learning, teaching and assessing: A revision of Bloom's Taxonomy of educational objectives: Complete edition. 2001, Longman: New York.

[15] D.A. Kolb, Experiential Learning: Experience as a Source of Learning and Development. 1984, New Jersey: Prentice Hall.

[16] J. Mezirow, How Critical Reflection Triggers Transformative Learning, in Fostering Critical Reflection in Adulthood, J.M. Associates, Editor. 1990, Jossey-Bass: San Francisco.

[17] D.A. Schön, The reflective practitioner: How professionals think in action. 1983, New York: Basic Books.

[18] S.A. Petersen and M. Oliveira, Reflection Continuum Model for Supporting Reflection and Game-Based Learning at the Workplace, in Serious Games - Third Joint International Conference, JCSG 2017, M. Alcañiz, S. Göbel, M. Ma, M. Oliveira, J.B. Hauge, and T. Marsh, Editors. 2017, Springer. p. 224-234. https://doi.org/10.1007/978-3-319-70111-0_21

[19] Hydro's technology pilot at Karmøy in full production. 2018 June 2018 [cited 20183 November]; Available from: https://www.hydro.com/en/press-room/Archive/2018/hydrostechnology-pilot-at-karmoy-in-full-production/.

[20] I. Nonaka and H. Takeuchi, The knowledge creating company: how Japanese companies create the dynamics of innovation. 1995, New York: Oxford University Press. https://doi.org/10.1016/0024-6301(96)81509-3

[21] J. Lave and E. Wenger, Situated Learning: Legitimate Peripheral Participation. 1991: Cambridge University Press. https://doi.org/10.1017/CBO9780511815355

[22] N. Suttie, S. Louchart, T. Lim, A. Macvean, W. Westera, D. Brown, and D. Djouti. Introducing the "Serious Games Mechanics": A Theoretical Framework to Analyse Relationships between "Game" and Pedagogical Aspects" of Serious Games. 2005; Available from: http://www.ludoscience.com/EN/diffusion/637-Introducing-the-Serious-GamesMechanics-A-Theoretical-Framework-to-Analyse-Relationships-Between-Game-andPedagogical-Aspects-of-Serious-Games.html.

[23] E.B.-N. Sanders and P.J. Stappers, Co-creation and the new landscapes of design. CoDesign International Journal of CoCreation in Design and the Arts, 2008. 4(1). https://doi.org/10.1080/15710880701875068 
pag. 56

[24] B. Crandall, G. Klein, and R. Hoffman, Working minds: A practitioner's guide to cognitive task analysis. 2006: MIT Press. https://doi.org/10.7551/mitpress/7304.001.0001

[25] G. Klein, Sources of power: How people make decisions. 1998, Cambridge, MA: MIT Press.

[26] L.G. Militello, R.B.J. Hutton, R.M. Pliske, B.J. Knight, and G. Klein, Applied Cognitive Task Analysis (ACTA) Methodology. 1997, Navy Personnel Research and Development Centre: San Dego, California. https://doi.org/10.1037/e443382005-001

[27] S.A. Petersen and M. Oliveira, The use of Reflection Continuum Model to support Digital Game-Based Learning for the development of Cognitive Skills in 11th European Conference on Game-Based Learning (ECGBL 2017). 2017: Graz, Austria. https://doi.org/10.1007/978-3319-70111-0_21

[28] I. Mayer, Bekebrede, G., C. Harteveld, H. Warmelink, J. Zhou, T. van Ruijven, J. Lo, R. Kortmann, and I. Wenzler, The research and evaluation of serious games: Toward a comprehensive methodology. British Journal of Educational Technology 2014. 45(3). https://doi.org/10.1111/bjet.12067 